Research Paper

\title{
Effects of Lipid Emulsion and Multivitamins on the Growth of Microorganisms in Peripheral Parenteral Nutrition Solutions
}

\author{
Takashi Kuwahara1, $^{\boxplus}$, Shinya Kaneda ${ }^{1}, K^{2}$ azuyuki Shimono ${ }^{1}$, Yoshifumi Inoue $^{2}$ \\ 1. Research and Development Center, Otsuka Pharmaceutical Factory, Inc., 115 Tateiwa, Naruto, Tokushima 772-8601, Japan; \\ 2. Center for Advanced Medical Engineering and Informatics, Osaka University, 2-2 Yamadaoka, Suita, Osaka 565-0871, Japan.
}

$\triangle$ Corresponding author: Takashi Kuwahara, Ph.D. Address: Research and Development Center, Otsuka Pharmaceutical Factory, Inc. 115 Tateiwa, Naruto, Tokushima 772-8601, Japan. Telephone: +81 886851151 (Ext. 2678) Fax: +81 $88 \quad 684 \quad 0553$ E-mail: Kuwahara.Takashi@otsuka.jp.

( ) Ivyspring International Publisher. This is an open-access article distributed under the terms of the Creative Commons License (http://creativecommons.org/ licenses/by-nc-nd/3.0/). Reproduction is permitted for personal, noncommercial use, provided that the article is in whole, unmodified, and properly cited.

Received: 2013.04.05; Accepted: 2013.06.14; Published: 2013.06.26

\begin{abstract}
Background: Blood stream infections caused by Bacillus cereus or Serratia marcescens in patients receiving peripheral parenteral nutrition (PPN) have occasionally been reported in Japan, but these microorganisms are not major causes of blood stream infections in patients receiving total parenteral nutrition via a central venous catheter. In Japan, commercially available PPN solutions contain amino acids, glucose, and electrolytes, but not contain lipid emulsion (LE) and multivitamins (MV). In this study, the effects of LE and MV on the growth of microorganisms such as Bacillus cereus, Serratia marcescens, Staphylococcus aureus, and Candida albicans in PPN solutions were investigated. Methods: A commercial 3\% amino acid and $7.5 \%$ glucose solution with electrolytes (AF) was used as the base solution to prepare test solutions (LAF, AFV, and LAFV) containing LE, MV, or both. Specifically, 20\% LE was added to AF in a ratio of I:9 to prepare LAF. MV was added to $A F$ and LAF to prepare AFV and LAFV, respectively. A specified number of each microorganism was added to each $100 \mathrm{~mL}$ of AF, LAF, AFV, and LAFV in sterile plastic flasks, and all flasks were allowed to stand at room temperature. The number of colony forming units per $\mathrm{mL}$ of each microorganism was counted at 0,24 , and 48 hours after the addition of each microorganism. Results: Both Bacillus cereus and Serratia marcescens increased rapidly in AF as well as in LAF, AFV, and LAFV. Staphylococcus aureus did not increased in AF, but increased slightly in LAF and increased rapidly in AFV and LAFV. Candida albicans increased slightly in AF and increased rapidly in LAF, AFV, and LAFV. Conclusions: The results suggest the followings: if microbial contamination occurs, I) Bacillus cereus and Serratia marcescens can grow rapidly in PPN solutions consisting of amino acids, glucose and electrolytes; 2) Staphylococcus aureus cannot grow without LE and MV, but can grow rapidly with MV; 3 ) Candida albicans can grow slowly without LE and MV, and the addition of LE or MV accelerates its growth.
\end{abstract}

Key words: microbial growth, parenteral nutrition, lipid emulsion, multivitamins, PPN, BSI.

\section{INTRODUCTION}

Peripheral parenteral nutrition (PPN) is regarded as a preferred alternative to short-term total parenteral nutrition via central vein (TPN) for many patients, because PPN avoids problems with central venous catheterization, simplifies nursing care, and reduces cost. ${ }^{1,2}$ In Japan, PPN is usually indicated for patients who require 1-week or shorter parenteral nutrition, and commercial PPN solutions contain 
amino acids, glucose, and electrolytes, but not contain lipid emulsion (LE) and multivitamins (MV). Since conventional short peripheral catheters are commonly used for PPN and midline catheters or fine-bore long catheters are rarely used in Japan, the $\mathrm{pH}$ values of commercial PPN solutions are designed to be greater than 6.5 to prevent infusion phlebitis.3,4 In other words, such physiological $\mathrm{pH}$ of PPN solutions are inevitably suitable for bacterial growth and PPN solutions may become a growth medium for bacterial species more than acidic TPN solutions.5,6 Recently, blood stream infection (BSI) outbreaks due to Bacillus cereus or Serratia marcescens in patients receiving PPN have occasionally been reported in Japan, ${ }^{7-10}$ but these microorganisms are not major causes of catheter-related blood stream infections (CRBSI) observed in TPN patients. In contrast, BSI due to gram positive cocci such as Staphylococcus aureus and Staphylococcus epidermidis, which are most frequent causes of CRBSI in TPN patients, ${ }^{11-13}$ has rarely been reported in PPN patients. In the present study, we investigated the effect of adding LE or/and MV to PPN solutions on the growth of the microorganisms; ie, Bacillus cereus as a delegate of gram positive rods, Serratia marcescens as a delegate of gram negative rods, Staphylococcus aureus as a delegate of gram positive cocci, and Candida albicans as a delegate of fungi.

\section{MATERIALS AND METHODS}

\section{Microorganisms employed}

A standard American Type Culture Collection (ATCC) strain and 2 clinical isolates were used for each microorganism, which were the standard strain ATCC11778 and the clinical isolates H-1 and H-2 of Bacillus cereus, the standard strain ATCC13880 and the clinical isolates N-4 and N-5 of Serratia marcescens, the standard strain ATCC6538 and the clinical isolates N-2 and N-3 of Staphylococcus aureus, and the standard strain ATCC10231 and the clinical isolates N-6 and N-7 of Candida albicans.

\section{Test solutions}

A commercial 3\% amino acid and 7.5\% glucose solution with electrolytes (AF: AMINOFLUID, Otsuka Pharmaceutical Factory, Inc., Japan), a commercial $20 \%$ LE preparation (Intralipos 20\%, Otsuka Pharmaceutical Factory, Inc.), and a commercial MV preparation (Otsuka MV Injection, Otsuka Pharmaceutical Factory, Inc.) were used. The compositions of AF, Intralipos 20\%, and Otsuka MV Injection are shown in Table 1, 2, and 3, respectively. Test solutions (LAF, $\mathrm{AFV}$, and LAFV) were prepared as follows. AF was used as the base solution, and 20\% LE was added to $\mathrm{AF}$ in a ratio of $1: 9$ to prepare LAF. To prepare AFV and LAFV, 1/20 of daily dose of MV was added to 100 $\mathrm{mL}$ of AF and LAF, respectively.

Table I. Composition of AF (AMINOFLUID).

\begin{tabular}{|c|c|c|c|}
\hline \multicolumn{4}{|c|}{ Composition per $1000 \mathrm{~mL}$} \\
\hline L-Leucine & $4.20 \mathrm{~g}$ & Total amino acids & $30.0 \mathrm{~g}$ \\
\hline L-Isoleucine & $2.40 \mathrm{~g}$ & Glucose & $75.0 \mathrm{~g}$ \\
\hline L-Valine & $2.40 \mathrm{~g}$ & $\mathrm{Na}^{+}$ & $35 \mathrm{mEq}$ \\
\hline L-Lysine $\mathrm{HCl}$ & $3.93 \mathrm{~g}$ & $\mathrm{~K}^{+}$ & $20 \mathrm{mEq}$ \\
\hline (as L-Lysine) & $(3.15 \mathrm{~g})$ & $\mathrm{Mg}^{2+}$ & $5 \mathrm{mEq}$ \\
\hline L-Threonine & $1.71 \mathrm{~g}$ & $\mathrm{Ca}^{2+}$ & $5 \mathrm{mEq}$ \\
\hline L-Tryptophan & $0.60 \mathrm{~g}$ & $\mathrm{Cl}^{-}$ & $35 \mathrm{mEq}$ \\
\hline L-Methionine & $1.17 \mathrm{~g}$ & $\mathrm{SO}_{4}{ }^{2-}$ & $5 \mathrm{mEq}$ \\
\hline L-Cysteine & $0.30 \mathrm{~g}$ & Acetate $^{-*}$ & $13 \mathrm{mEq}$ \\
\hline L-Phenylalanine & $2.10 \mathrm{~g}$ & Gluconate $^{-}$ & $5 \mathrm{mEq}$ \\
\hline L-Tyrosine & $0.15 \mathrm{~g}$ & L-Lactate ${ }^{-}$ & $20 \mathrm{mEq}$ \\
\hline L-Arginine & $3.15 \mathrm{~g}$ & Citrate ${ }^{3-*}$ & $6 \mathrm{mEq}$ \\
\hline L-Histidine & $1.50 \mathrm{~g}$ & $\mathrm{P}$ & $10 \mathrm{mmol}$ \\
\hline L-Alanine & $2.40 \mathrm{~g}$ & $\mathrm{Zn}$ & $5 \mu \mathrm{mol}$ \\
\hline L-Proline & $1.50 \mathrm{~g}$ & \multicolumn{2}{|c|}{ Characteristics } \\
\hline L-Serine & $0.90 \mathrm{~g}$ & $\mathrm{pH}$ & 6.6 \\
\hline Glycine & $1.77 \mathrm{~g}$ & Titratable acidity & $8.4 \mathrm{mEq} / \mathrm{L}$ \\
\hline L-Aspartic acid & $0.30 \mathrm{~g}$ & Osmolality & $856 \mathrm{mOsm} / \mathrm{kg}$ \\
\hline L-Glutamic acid & $0.30 \mathrm{~g}$ & OPR & Approximately 3 \\
\hline
\end{tabular}

*, including the amount derived from the additives. OPR, osmotic pressure ratio to physiological saline. 
Table 2. Composition of Intralipos $20 \%$.

\begin{tabular}{lc}
\hline \multicolumn{2}{c}{ Composition per $100 \mathrm{~mL}$} \\
\hline Soybean oil & $20.0 \mathrm{~g}$ \\
Egg yolk lecithin & $1.2 \mathrm{~g}$ \\
Glycerol & $2.2 \mathrm{~g}$ \\
\hline \multicolumn{2}{c}{ Characteristics } \\
\hline $\mathrm{pH}$ & 7.2 \\
Osmolality & $284 \mathrm{mOsm} / \mathrm{kg}$ \\
\hline
\end{tabular}

Table 3. Composition of Otsuka MV Injection.

\begin{tabular}{|c|c|}
\hline \multicolumn{2}{|c|}{ Composition per $4 \mathrm{~mL}$ (daily dose) } \\
\hline Vitamin $\mathrm{B}_{1}$ & $3.1 \mathrm{mg}$ \\
\hline Vitamin $B_{2}$ & $3.6 \mathrm{mg}$ \\
\hline Vitamin $\mathrm{B}_{6}$ & $4.0 \mathrm{mg}$ \\
\hline Vitamin $\mathrm{B}_{12}$ & $0.005 \mathrm{mg}$ \\
\hline Vitamin C & $100 \mathrm{mg}$ \\
\hline Folic acid & $0.4 \mathrm{mg}$ \\
\hline Nicotinamide & $40 \mathrm{mg}$ \\
\hline Biotin & $0.06 \mathrm{mg}$ \\
\hline Panthenol & $14 \mathrm{mg}$ \\
\hline Vitamin A oil & 3300 Vit.A IU \\
\hline Vitamin $\mathrm{D}_{3}$ & $0.005 \mathrm{mg}$ \\
\hline Vitamin E & $10 \mathrm{mg}$ \\
\hline Vitamin K & $2 \mathrm{mg}$ \\
\hline
\end{tabular}

Vit.A IU, international unit for vitamin A.

\section{Addition of microorganism, incubation, and sampling}

A specified number of each test microorganism was added to each $100 \mathrm{~mL}$ of test solutions in sterile plastic flasks, and all flasks were allowed to stand at room temperature $\left(23-26^{\circ} \mathrm{C}\right)$. An aliquot $(3-25 \mathrm{~mL})$ of test solution was sampled at 0,24 , and 48 hours after the addition of microorganism. The aliquot for 0 hour was sampled a few minute after the addition of microorganism, and the values obtained at 0 hour show the added amount of each microorganism.

\section{Measurement of viable cells}

Each aliquot of test solution sampled was inoculated in a Soybean Casein Digest (SCD) agar plate in duplicate. When necessary, the test solution sampled was diluted 10 -fold to $10^{7}$-fold with physiological saline before inoculation. When 1 to $10 \mathrm{~mL}$ of test solution was examined, the solution was filtered with a membrane filter $(0.45 \mu \mathrm{m}$, nitrocellulose) for the sterility test of Japanese Pharmacopoeia, and the filter was put on a SCD agar plate. After 12 to 24 hours of incubation at $37^{\circ} \mathrm{C}$, the number of colony forming units (CFU) of each microorganism was counted for each plate, and the mean CFU of duplicate data was calculated. The number of CFU per $\mathrm{mL}$ was calculated by using the number of CFU per plate, inoculated volume, and diluting ratio. The results are shown as values of $\mathrm{CFU} / \mathrm{mL}$ in semi-logarithmic graphs. Same as other experimental studies of microbial growth, ${ }^{14-18}$ the data obtained in this study were not analyzed statistically because biological significance of these kinds of data is considered assessable without statistical analysis.

\section{RESULTS}

\section{Bacillus cereus (Figure I)}

All 3 strains of B. cereus increased rapidly in all of $\mathrm{AF}, \mathrm{LAF}, \mathrm{AFV}$, and $\mathrm{LAFV}$, reaching the maximum concentration within 48 hours. At 24 hours, the addition of LE or MV accelerated the proliferation.

\section{Serratia marcescens (Figure 2)}

All 3 strains of $S$. marcescens increased rapidly in all test solutions, $\mathrm{AF}, \mathrm{LAF}, \mathrm{AFV}$, and $\mathrm{LAFV}$, reaching the maximum concentration within 48 hours. At 24 hours, the addition of LE accelerated the proliferation.

\section{Staphylococcus aureus (Figure 3)}

In $\mathrm{AF}$, all 3 strains of $S$. aureus did not increase for 48 hours, and 2 clinical isolates were not visible after 24 hours. In LAF, a clinical isolate N-2 did not increase, while the other 2 strains increased slightly. Also in AFV and LAFV, N-2 did not increase for 48 hours. However, the other 2 strains (the standard strain ATCC6538 and the clinical isolate N-3) increased rapidly in AFV and LAFV and almost reached the maximum concentration.

\section{Candida albicans (Figure 4)}

All 3 strains of $C$. albicans increased slightly in $\mathrm{AF}$, but increased rapidly in LAF, AFV, and LAFV.

\section{DISCUSSION}

Catheter-related blood stream infection (CRBSI) is one of the most common complications of intravenous catheters. ${ }^{19-22}$ For microorganisms, Staphylococcus aureus, coagulase-negative staphylococci, Candida albicans, Escherichia coli, Klebsiella pneumonia, etc. have been shown as the major causes of CRBSI in patients receiving total parenteral nutrition via central vein (TPN). ${ }^{11-13}$ Recently, blood stream infection (BSI) outbreaks caused by Bacillus cereus or Serratia marcescens in patients receiving peripheral parenteral nutrition (PPN) have occasionally been reported in Japan, 7-10 but these microorganisms are not major causes of CRBSI in TPN patients. In contrast, BSI due to gram positive cocci such as Staphylococcus aureus or Staphylococcus epidermidis has rarely been reported in PPN patients. In the present study, we investigated the possibility of growth of microorganisms such as $\mathrm{Ba}$ - 
cillus cereus, Serratia marcescens, Staphylococcus aureus, and Candida albicans in PPN solutions. In particular, the effects of the addition of lipid emulsion (LE) and

\section{Bacillus cereus}

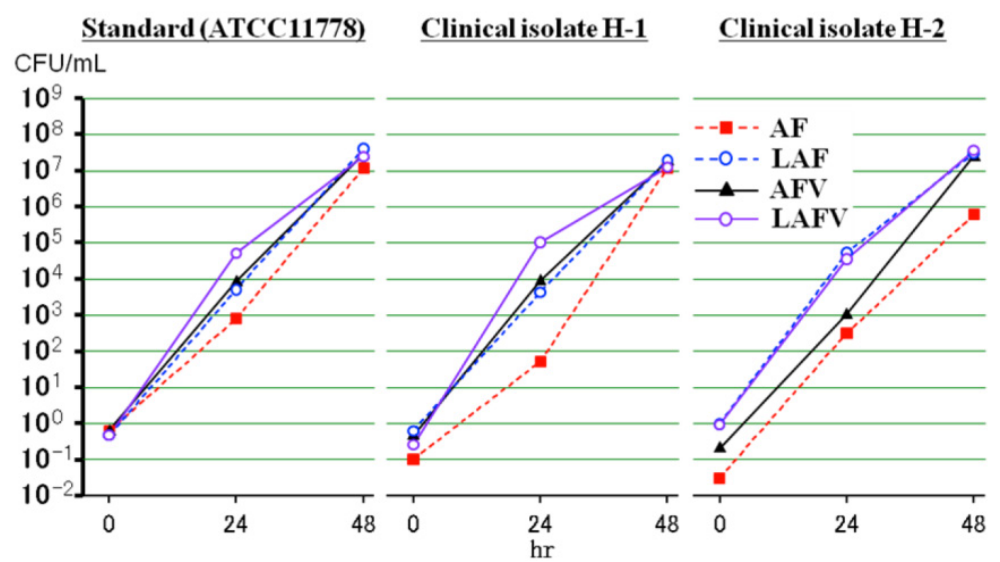

Figure I. Effect of lipid emulsion or/and multivitamins on the growth of Bacillus cereus in $\mathrm{AF}$ ( $\mathrm{pH}$ is 6.6 and OPR is approximately 3). LAF was prepared by mixing $A F$ and $20 \% \mathrm{LE}$ in a ratio of 9:I. AFV or LAFV was prepared by adding $1 / 20$ of daily dose of MV to $100 \mathrm{~mL}$ of AF or LAF. OPR, osmotic pressure ratio to physiological saline.

\section{Serratia marcescens}

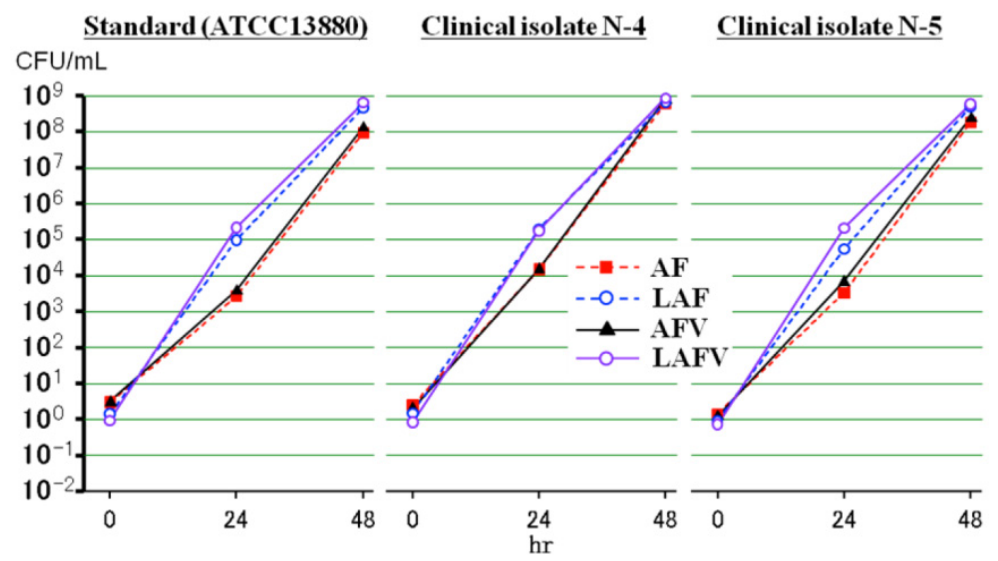

Figure 2. Effect of lipid emulsion or/and multivitamins on the growth of Serratia marcescens in AF ( $\mathrm{pH}$ is 6.6 and OPR is approximately 3). LAF was prepared by mixing $A F$ and $20 \% \mathrm{LE}$ in a ratio of 9:I. AFV or LAFV was prepared by adding $1 / 20$ of daily dose of $M V$ to $100 \mathrm{~mL}$ of AF or LAF. OPR, osmotic pressure ratio to physiological saline.

\section{Staphylococcus aureus}

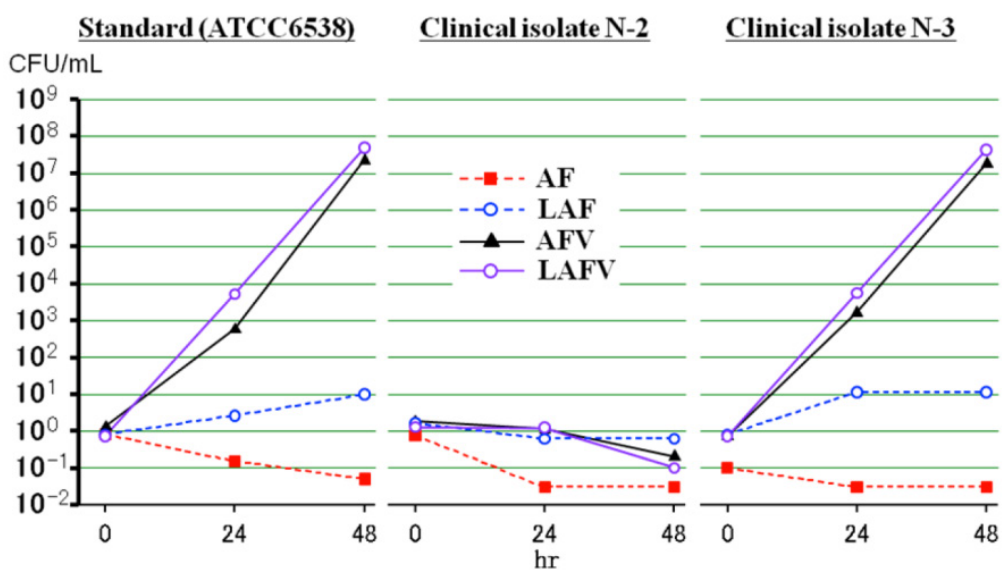

Figure 3. Effect of lipid emulsion or/and multivitamins on the growth of Staphylococcus aureus in AF (pH is 6.6 and OPR is approximately 3). LAF was prepared by mixing AF and $20 \% \mathrm{LE}$ in a ratio of 9:I. AFV or LAFV was prepared by adding $1 / 20$ of daily dose of MV to I00 mL of AF or LAF. OPR, osmotic pressure ratio to physiological saline. 


\section{Candida albicans}

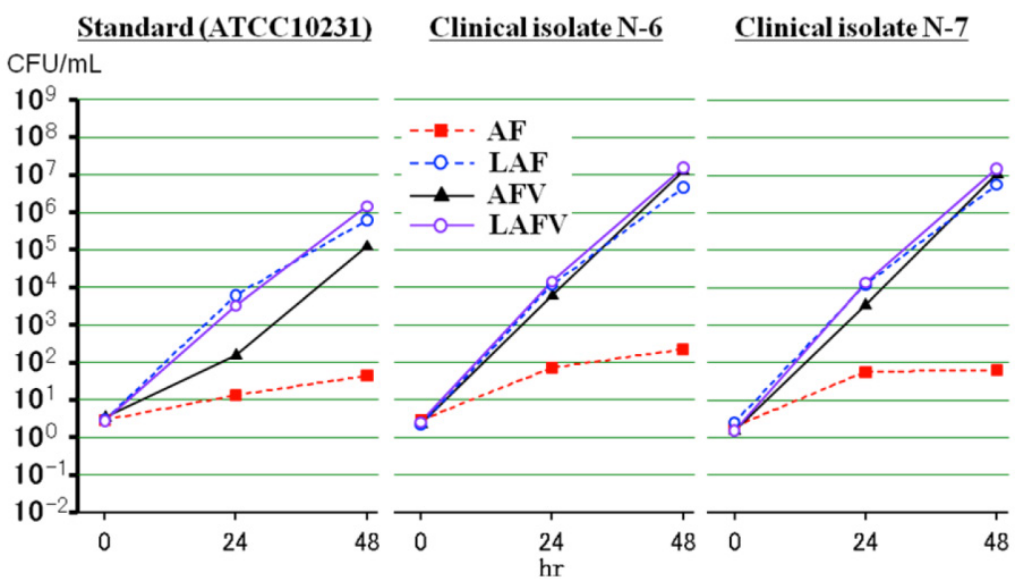

Figure 4. Effect of lipid emulsion or/and multivitamins on the growth of Candida albicans in AF ( $\mathrm{pH}$ is 6.6 and OPR is approximately 3). LAF was prepared by mixing $A F$ and $20 \% \mathrm{LE}$ in a ratio of 9:I. AFV or LAFV was prepared by adding I/20 of daily dose of MV to $100 \mathrm{~mL}$ of AF or LAF. OPR, osmotic pressure ratio to physiological saline.

In the study, a commercial 3\% amino acid and $7.5 \%$ glucose solution with electrolytes (AF) was used as the base solution, and AF was added with LE, MV, or both. A specified number of each microorganism was added to each $100 \mathrm{~mL}$ of test solutions in sterile plastic flasks. The number of colony forming units $(\mathrm{CFU}) / \mathrm{mL}$ of each microorganism was counted at 0 , 24 , and 48 hours after the addition of microorganism. All 3 strains of $B$. cereus increased rapidly in all test solutions, and the addition of LE or MV seemed to accelerate the proliferation. This finding indicates that $B$. cereus can increase rapidly in PPN solutions without LE and MV, and the addition of LE or MV accelerates the growth. All 3 strains of $S$. marcescens increased rapidly in all test solutions, and the addition of LE seemed to accelerate the proliferation. Thus, it is indicated that $S$. marcescens also can increase rapidly in PPN solutions without LE and MV and the addition of LE accelerates the growth. All 3 strains of $S$. aureus did not increase for 48 hours in AF without LE and $\mathrm{MV}$, and 1 clinical isolate did not increase in $\mathrm{AF}$ added with LE, MV, or both. However, the other 2 strains increased slightly in AF added with LE, increasing rapidly in $\mathrm{AF}$ added with $\mathrm{MV}$ and in $\mathrm{AF}$ added with both LE and MV. These results indicate that $S$. aureus cannot increase in PPN solutions without LE and MV, but some strains may increase rapidly by adding MV. Since 2 of the 3 strains increased rapidly with no increase in 1 strain, there seems to be a strain difference in auxotrophy of $S$. aureus. All 3 strains of $C$. albicans increased slightly in AF without $\mathrm{LE}$ and MV, but increased rapidly in AF added with $\mathrm{LE}, \mathrm{MV}$, or both, indicating that $C$. albicans increases slightly in PPN solutions without LE and MV and the addition of LE or MV accelerates the growth. These results show that $B$. cereus and $S$. marcescens can grow rapidly in PPN solutions containing amino acids, glucose and electrolytes, but $S$. aureus cannot grow similarly.

We have previously shown that adding water-soluble vitamins does not affect the growth of $S$. aureus but water-insoluble vitamins are essential for the growth of S. aureus. ${ }^{23}$ PPN solutions available in Japan consist of amino acids, glucose and electrolytes but do not contain LE and MV, although the latest PPN products contain only thiamine $\left(B_{1}\right)$ for the prevention of Wernicke's encephalopathy or lactic acidosis. In clinical practice, PPN solutions are sometimes supplemented with water-soluble vitamins such as $B_{1}$, $B_{2}, B_{6}, B_{12}$, and $C$, but rarely supplemented with MV including water-insoluble vitamins. With regard to the $\mathrm{pH}$ value of PPN solutions, the physiological $\mathrm{pH}$ of Japanese PPN solutions seems to be suitable for bacterial growth, 5,6 and B. cereus and S. marcescens can increase rapidly without LE and MV. With regard to BSI, cases with B. cereus and S. marcescens have been reported in PPN patients, while cases with $S$. aureus, perhaps also with $S$. epidermidis, have scarcely been reported in PPN patients. This is likely because those cannot increase without LE and water-insoluble vitamins. On the other hand, commercially available PPN solutions in European countries and some other countries are acidic ( $\mathrm{pH} 5-6)^{2,24,25}$ as similar to TPN solutions, and then BSI outbreaks by B. cereus or $S$. marcescens have rarely been reported. However, PPN and TPN solutions with physiological $\mathrm{pH}$ have recently been launched in European countries, ${ }^{26}$ and these solutions contain LE and are usually supplemented with MV, which would be suitable for the growth of all the microorganisms employed in the present study.

Taken together, the results of this study suggest 
the followings: if microbial contamination occurs, 1) Bacillus cereus and Serratia marcescens can grow rapidly in PPN solutions consisting of amino acids, glucose and electrolytes; 2) Staphylococcus aureus cannot grow without LE and MV, but can grow rapidly with MV; 3) Candida albicans can grow slightly without LE and $\mathrm{MV}$, and the addition of LE or MV accelerates its growth. Therefore, it is strongly recommended that even in PPN, nutrient solutions must be prepared under strict aseptic conditions and procedures, and intravenous lines must be cared properly.

\section{ACKNOWLEDGEMENTS}

We are very grateful to Masao Ichihara for his helpful suggestion.

\section{ABBREVIATIONS}

AF: a commercial 3\% amino acid and $7.5 \%$ glucose solution with electrolytes (AMINOFLUID, Otsuka Pharmaceutical Factory, Inc., Japan); AFV: a test solution prepared by adding $1 / 20$ of daily dose of multivitamins to $100 \mathrm{~mL}$ of AF; ATCC: American Type Culture Collection; BSI: blood stream infection; CFU: colony forming units; CRBSI: catheter-related blood stream infections; LAF: a test solution prepared by mixing AF and $20 \%$ lipid emulsion in a ratio of $9: 1$; LAFV: a test solution prepared by adding $1 / 20$ of daily dose of multivitamins to $100 \mathrm{~mL}$ of LAF; LE: lipid emulsion; MV: multivitamins; OPR: osmotic pressure ratio to physiological saline; PPN: peripheral parenteral nutrition; SCD: Soybean Casein Digest; TPN: total parenteral nutrition via central vein; Vit.A IU: international unit for vitamin A.

\section{COMPETING INTERESTS}

TK, SK and KS are employees for Otsuka Pharmaceutical Factory, inc. YI has declared that no competing interest exists.

\section{References}

1. Payne-James JJ, Khawaja HT. First choice for total parenteral nutrition: the peripheral route. J Parenter Enteral Nutr. 1993;17:468-478.

2. Anderson ADG, Palmer D, MacFie J. Peripheral parenteral nutrition. Br J Surg. 2003;90:1048-1054.

3. Kuwahara T, Asanami S, Kawauchi Y, Kubo S. Experimental infusion phlebitis: tolerance $\mathrm{pH}$ of peripheral vein. J Toxicol Sci. 1999;24:113-121.

4. Kuwahara T, Kaneda S, Sawamoto O, Kohno E. Cyclic infusion is effective in reducing phlebitis caused by peripheral parenteral nutrition solution: an experimental study in rabbits. e-SPEN. 2009;4:e344-e347.

5. Kuwahara T, Kaneda S, Shimono K, Inoue Y. Growth of microorganisms in total parenteral nutrition solutions without lipid. Int J Med Sci. 2010;7:43-47.

6. Kuwahara T, Shimono K, Kaneda S, Tamura T, Ichihara M, Nakashima Y. Growth of microorganisms in total parenteral nutrition solutions containing lipid. Int J Med Sci. 2010;7:101-109.

7. Sasahara T, Hayashi S, Morisawa Y, Sakihama T, Yoshimura A, Hirai Y. Bacillus cereus bacteremia outbreak due to contaminated hospital linens. Eur J Clin Microbiol Infect Dis. 2011;30:219-226.
8. Aso Y, Nagatomi M, Nakazawa T, Sakaki S, Ishi K. Examination of infection fluid type and environmental factors involved in increased $\mathrm{Ba}$ cillus cereus bloodstream infection. Jpn J Environ Infect. 2012;27:81-90. (in Japanese).

9. Matsumoto S, Suenaga H, Naito K, Sawazaki M, Hiramatsu T, Agata N. Management of suspected nosocomial infection: an adult of 19 hospitalized patients with septicemia caused by Bacillus species. Jpn J Infect Dis. 2000;53:196-202.

10. Iwaya A, Nakagawa S, Taneike I, et al. Serratia infection and sanitary management of fluid administration. Niigata-igakukai-zasshi 2003;117:469-478. (in Japanese)

11. Llop J, Badia MB, Comas D, Tubau M, Jodar R. Colonization and bacteremia risk factors in parenteral nutrition catheterization. Clin Nutr. 2001;20:527-534.

12. Hojsak I, Strizic H, Misak Z, et al. Central venous catheter related sepsis in children on parenteral nutrition: A 21-year single-center experience. Clin Nutr. 2012;31:672-675.

13. Dreesen M, Foulon V, Spriet I, et al. Epidemiology of catheter-related infections in adult patients receiving home parenteral nutrition: A systematic review. Clin Nutr. 2013;32:16-26.

14. Didier ME, Fischer S, Maki DG. Total nutrient admixtures appear safer than lipid emulsion alone as regards microbial contamination: growth properties of microbial pathogens at room temperature. J Parenter Enteral Nutr. 1998;22:291-296.

15. Jarvis WR, Highsmith AK. Bacterial growth and endotoxin production in lipid emulsion. J Clin Microbiol. 1984;19:17-20.

16. Keammerer D, Mayhall CG, Hall GO, Pesko LJ, Thomas RB. Microbial growth patterns in intravenous fat emulsions. Am J Hosp Pharm. 1983;40:1650-1653.

17. Murano A, Morinaga N, Iwamaru Y, et al. Acidic conditions enhance bactericidal effects of sodium bisulfite on Helicobacter pylori. Helicobacter 2005;10:132-135.

18. Obayashi A, Oie S, Kamiya A. Microbial viability in preparations packaged for single use. Biol Pharm Bull. 2003;26:667-670.

19. Mermel LA, Farr BM, Sherertz RJ, et al. Guidelines for the management of intravascular catheter-related infection. Clin Infect Dis. 2001;32:1249-1272.

20. Banton J. Techniques to prevent central venous catheter infection: products, research, and recommendations. Nutr Clin Pract. 2006;21:56-61.

21. Kruse JA, Shah NJ. Detection and prevention of central venous catheter-related infections. Nutr Clin Pract. 1993;8:163-170.

22. Freund HR, Rimon B. Sepsis during total parenteral nutrition. J Parenter Enteral Nutr. 1990;14:39-41.

23. Shimono K, Kaneda S, Kuwahara T, Kawaguchi Y, Momii A. Effects of multivitamins on the growth of microorganisms in peripheral parenteral nutrition solutions [abstract]. Clin Nutr. 2005;24:707.

24. Baxter International Inc. OliClinomel. Product brochure.

25. Fresenius Kabi Deutschland $\mathrm{GmbH}$. StructoKabiven and StructoKabiven Peripheral. Product brochure.

26. Baxter International Inc. OLIMEL. Product brochure. 